\title{
Revisiting the Ethical Aspects in Research Publications
}

\section{Asama Mukherjee}

Department of Gynaecological Oncology, Tata Medical Center, Newtown, Kolkata, West Bengal, India.

\begin{abstract}
Increasing peer-pressure for publishing research articles accounts for the several instances of misconducts in the form of fabrication, falsification, plagiarism, salami slicing and authorship related issues. Advancement in technology has not only enabled the process of speedy publications but also the detection of unethical practices. Instances of paper retraction and suspension of authors is leading to loss of trust among researchers. A continuous training on research ethics needs to be encouraged from the level of universities and research institutes. This article aims to imbibe a sense of responsibility among authors, reviewers, editors and publishers in making correct ethical decision in research publication in order to achieve a healthy research outcome.
\end{abstract}

Key words: Ethics in research, research misconducts, Committee on Publication Ethics.

\section{Introduction}

To 'publish or perish' has been the keynote for professional existence of many academicians. Publications are the index to measure a researcher's accomplishments, for assessing productivity, evaluate-ng credits for their contributions in a specific field and drive to take up innovative projects. They are frequently important in the current scenario for recruitment and appraisal process or achieving a prestigious grant or thrive in a competitive research environment. Consequently, there has been an explosion in publishing in the last few decades. Researchers are in a rat-race for getting their papers published in high impact

Address of Correspondence: Dr. Asama Mukherjee, Department of Gynaecological Oncology, Tata Medical Center, Newtown, Kolkata-700156, West Bengal, India. Email id: asamamukherjee@yahoo.com (Received 07 January 2020; revised 16 January 2020; accepted 18 January 2020). journals with high citation and h-index. Research in any discipline is expected to be transparent, unbiased and unambiguous. Their communications are expected to be honest declarations of their work. In this perspective, it is therefore extremely relevant to discuss and revisit the agenda of ethics (1) in publishing research work.

The burden of scientific data has been ever-increasing. Digitalisation has completely transformed the publication industry. It has paved the way for meeting demands of authors for communication in a faster and effective way, which can be widely reached by the community and that too with the longest possible lifespan. The hallmark of publishing an original research article is the trust between authors, reviewers and editors. Each of them is expected to abide by certain ethical principles for the communications. While authors are primarily responsible for sharing authentic data 
that are accountable, reproducible and credible, with a broader aim that is beneficial to the community, reviewers and editors are also expected to uphold the ethical code of conduct for the process of publication of the valuable piece of research following a fair peer-review process. However, the pressure to increase the number of publications for meeting professional requirements have led to unethical practises or misconducts $(2$, 3 ) that are being frequently reported. Common forms of research misconduct includes fabrication, falsification, plagiarism, salami slicing as well as issues related to authorship, including misrepresentation of author's institutional affiliation.

Most academic publishers have developed their publishing policies and standards based on the guidance of the Committee on Publication Ethics (COPE) (4) on the common ethical issues. In order to detect plagiarism, several softwares tools like Turnitin, Paperrater, Dupli Checker, Copy Leaks, Plagiarism etc. are in use. Several other screening tools for quality assurance and detection of frauds images are under development (5). Although there are still several loop holes and inadequacies in the process, which has made the job of editors and reviewers really tedious! Authorship issues are even more complex. The International Committee of Medical Journal Editors (ICMJE) had laid down policies for authorship (6) in a publication which states that it is to be given to those who have substantial intellectual contribution to a research work. Moreover, the listed authors should essentially understand their role in taking responsibility and be accountable for the publication. However, the concept of authorship is most often being misused. The practise of gift, honorary, and guest authorship have infiltrated largely for opportunistic reason. Ghost authorship is misconduct not only from ethical but also from professional standpoint. The Office of Research Integrity (ORI) under US Department of Health and Human Services is a body that oversees and directs the integrity of Public Health Service (PHS) research activities by developing policies, procedures and regulations related to the detection, investigation, and prevention of re- search misconduct. The ORI recommends the use of some 'forensic tools' (7) for examination of questionable images. One such type is 'forensic droplets'. The Division of Investigative Oversight (DIO) of the ORI commonly uses Adobe Bridge and Image $\mathrm{J}$ for monitoring fraud in images.

Ethical misconduct has far reaching effects. Depending upon the policies of the publisher, it leads to paper retraction and suspension of authors. This marks a negative impact on researchers. The worst repercussion of it is perhaps loss of trust and confidence among the research community. Publications provide the source of knowledge and information in a given discipline. As researchers rely on published materials, it is their utmost responsibility to share quality knowledge with care. A recently published editorial by Francois-Xavier Coudert in the Chemistry of Material journal (8) reveals the retraction practises in chemistry and material science using information from Scopus database. The article presents some interesting data on retraction rate from different countries. The author has also identified the causes for retraction which is primarily plagiarism, issues related to authorship and data reporting.

Another report by Enrico M. Bucci detects image manipulations in published manuscripts using a software pipeline (9) that reveals a linear correlation between the retraction rate and the rate of manipulated images using data from PubMed Central and the post publication peer review website PubPeer. Paper retraction due to fake peer reviews have also been reported (10). The list of such instances in misconducts is inexhaustible.

\section{Conclusions}

It is now time to take a step forward to address this malaise. The misconduct-related matters should be brought to light involving open and frank discussion at the level of universities and research institutions. Students should be mentored continuously and awareness related to

\section{International Research Journal of Multidisciplinary Scope (IRJMS)}


responsible research practises should be conducted in institutions. Peer-monitoring among researchers that would enhance transparency, facilitate acceptance of shortcomings and finetuning of their research skills should be encouraged. A robust surveillance system consisting of software tools for efficiently detecting fraud or manipulate-on in images, data and figures should be developed to equip reviewers and editors tackle the situation. Stringent criteria related to authorship policies should also be imposed. Let us prevent erosion of moral values and encourage ourselves to make ethical decisions. Most importantly, let us enjoy research and aim publishing in quality not in quantity.

\section{References}

1. Sharma OP. Ethics in Science. Indian Journal of Microbiology. 2015; 55(3): 341-344.
2. Masic I. Plagiarism in Scientific Research and Publications and How to Prevent It. Mater Sociomed. 2014; 26(2): 141-146.

3. Mandal M, Bagchi D, Basu SR. Scientific misconducts and authorship conflicts: Indian perspective. Indian Journal of Anesthesia. 2015; 59(7): 400-405.

4. General approach to publication ethics for the Editoral Office. DOI: https://doi.org/10.24318/cope.2019.2. 24.

5. Koppers L, Wormer H, Ickstadt K. Towards a Systematic Screening Tool for Quality Assurance and Semiautomatic Fraud Detection for Images in the Life Sciences. Sci Eng Ethics. 2017; 23(4): 1113-1128.

6.http://www.icmje.org/recommendations/browse/ro les-and-responsibilities/defining-the-role-of-authorsand-contributors.html.

7. https://ori.hhs.gov/forensic-tools.

8. Coudert FX. Correcting the Scientific Record: Retraction Practices in Chemistry and Materials Science. Chem. Mater. 2019; 31(10): 3593-3598.

9. Bucci EM. Automatic detection of image manipulations in the biomedical literature. Cell Death \& Disease. 2018; 9: 400.

10. Qi X, Deng H, Guo X. Characteristics of retractions related to faked peer reviews: an overview. Postgraduate Medical Journal. 2016; 93(1102): 499-503. 